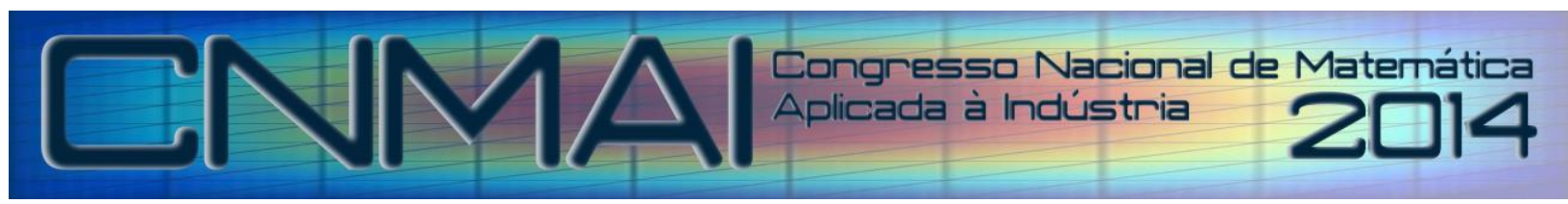

18 a 21 de novembro de 2014, Caldas Novas - Goiás

\title{
SIMULAÇÃO NUMÉRICO-COMPUTACIONAL DE PLACAS FINAS EM GRANDES DESLOCAMENTOS
}

\author{
Andressa Fernanda Rosa de Lima, andressafernanda@ hotmail.com.br ${ }^{1}$ \\ Antônio Marcos Gonçalves de Lima, amglima@ mecanica.ufu.br ${ }^{2}$ \\ Romes Antônio Borges, romes@ufg.br ${ }^{3}$
}

${ }^{1}$ Universidade Federal de Uberlândia - Faculdade de Engenharia Mecânica, Av. João Naves de Ávila 2121, 38408-100, Uberlândia, Minas Gerais, Brasil

${ }^{2}$ Universidade Federal de Uberlândia - Faculdade de Engenharia Mecânica, Av. João Naves de Ávila 2121, 38408-100, Uberlândia, Minas Gerais, Brasil

${ }^{3}$ Universidade Federal de Goiás - Campus Catalão - Departamento de Matemática e Matemática Industrial, Av. Dr. Lamartine Pinto de Avelar 1120, 75704-020, Catalão, Goiás, Brasil

Resumo:No meio industrial, a busca-se constantemente a confiabilidade, durabilidade e segurança das máquinas e equipamentos utilizados na atividade produtiva. Neste contexto, nas fases de concepção de projeto e análise são geralmente empregados procedimentos numérico-computacionais para prever o comportamento de sistemas mecânicos quando estes são submetidos a perturbações estáticas ou dinâmicas. Essas perturbações têm se tornado cada vez mais intensas enquanto os materiais utilizados na realização das estruturas são cada vez mais finos, leves e extensos. Portanto, devido a essas condições, ocorrem nesses sistemas comportamentos não-lineares, que podem resultar em falhas catastróficas quando esses fenômenos não são devidamente reconhecidos e estudos para serem controlados. Nesse trabalho, pretende-se implementar uma modelagem numérico-computacional para o estudo e previsão do comportamento de uma estrutura do tipo placa fina de interesse industrial, comumente utilizado em projetos da indústria aeronáutica e automobilística, submetidas a grandes deslocamentos, com o objetivo de obter as suas respostas devido a diferentes condições de operação.

Palavras-chave: Sistemas não-lineares, elementos finitos, modelagem

\section{INTRODUÇÃ̃}

Fenômenos não-lineares são muito comuns na engenharia prática, sendo os fenômenos lineares uma exceção. Devido ao comportamento imprevisível de sistemas não-lineares podem ocorrer falhas catastróficas As não-linearidades de estruturas podem ser consideradas locais ou globais; elas dependem do comportamento do material, das condições limite ou das condições de contorno.

As não-linearidades geométricas são frequentemente encontradas em sistemas dinâmicos estruturais em que a espessura é considerada fina. Nesses sistemas são feitas as seguintes considerações: a) As deformações são elásticas e infinitesimais; b) as rotações são moderadas. Tal comportamento é observado quando o deslocamento transversal é maior que a espessura da estrutura. (Gerges,2013).

Nesse contexto, podem ser incluídas as placas finas, que são extensamente utilizadas no meio industrial, como por exemplo: na indústria aeronáutica compõe a fuselagem e as asas do avião; na indústria automobilística podem ser utilizadas na construção da estrutura dos veículos.

Uma placa é considerada fina, caso a relação entre o seu comprimento e a espessura seja superior a 20. Estas estruturas são muito sensíveis à vibração, e quando são submetidas a grandes deslocamentos apresentam um comportamento nãolinear.

Para a modelagem de uma placa fina em grandes deslocamentos, assume-se o modelo de Love-Kirchhoff onde a partir dos campos de deslocamentos, obtêm-se as deformações da placa através do tensor de Green-Lagrange.

O que difere essa modelagem das convencionais, é o fato de que quando as placas finas estão submetidas à grandes deslocamentos, a modelagem não podem ser considerados os efeitos de flexão e deformações no plano da placa independentes. Deve-se também considerar os que estes efeitos são acoplados, de onde surgem as não-linearidades do sistema. (Zienkiewicz e Taylor, 2000).

Devido às características não-lineares da estrutura, a resolução da equação do movimento que rege o sistema tornase mais complexa, sendo assim, é utilizado o método de integração de Newmark, que permite a resolução de equações diferenciais de segunda ordem 
Nesse trabalho é realizada uma modelagem numérico-computacional de uma estrutura do tipo placa fina, aplicandose diferentes condições como por exemplo, amplitude da frequência de excitação e tipo de excitação, à estrutura e são realizadas análises das respostas visando o entendimento da influência desses parâmetros em um sistema não-linear.

\section{MODELAGEM DE PLACAS FINAS SUJEITAS A GRANDES DESLOCAMENTOS}

As estruturas do tipo placa fina, são imensamente utilizadas no meio industrial, e, devido a demanda por objetos cada vez mais leves e resistentes para serem operados em condições cada vez mais críticas, muitos pesquisadores têm se dedicado ao estudo do comportamento desses sistemas quando operados em situações que exigem muito da estrutura, como por exemplo, placas finas quando submetidas a grandes deslocamentos.

Considera-se como grandes deslocamentos, quando o mesmo ultrapassa a espessura da placa (Moussaoui e Benamar, 2002). E neste caso, a teoria de placas finas que adota as relações lineares entre tensão e deformação já não é mais suficiente para descrever o comportamento da estrutura e, nesse sentido, busca-se modelagens com o objetivo de se alcançar respostas que mostram as respostas o mais aproximado da realidade o possível.

Neste trabalho, é utilizado o modelo de Love-Kirchhoff que é inspirado no modelo de Euler-Bernoulli para placas, onde é utilizado o seguinte tensor de deformação de Green-Lagrange para a formulação do princípio variacional:

$$
\boldsymbol{\varepsilon}=\frac{1}{2}\left(\nabla \boldsymbol{U}+\nabla \boldsymbol{U}^{T}\right)+\frac{1}{2} \nabla \boldsymbol{U} \nabla \boldsymbol{U}^{T}
$$

Onde $\boldsymbol{U}$ é admitido como sendo a aproximação do campo de deslocamentos.

Admitindo a hipótese de pequenas deformações e de rotações moderadas, pode-se reescrever o tensor de GreenLagrange sob a seguinte forma:

$$
\boldsymbol{\varepsilon}=\left\{\begin{array}{c}
\varepsilon_{x x} \\
\varepsilon_{y y} \\
2 \gamma_{x y}
\end{array}\right\}=\underbrace{\left\{\begin{array}{c}
u_{x} \\
v_{y} \\
u_{y}+v_{x}
\end{array}\right\}}_{\boldsymbol{\varepsilon}^{m}}-z \underbrace{\left\{\begin{array}{c}
w_{x x} \\
w_{y y} \\
2 w_{x y}
\end{array}\right\}}_{\boldsymbol{K}^{f}}+\frac{1}{2} \underbrace{\left\{\begin{array}{c}
w_{x}^{2} \\
w_{y}^{2} \\
2 w_{x} w_{y}
\end{array}\right\}}_{\boldsymbol{\varepsilon}^{c}}
$$

onde $\boldsymbol{\varepsilon}^{m}$ e $\boldsymbol{\varepsilon}^{f}=z \boldsymbol{K}^{f}$ representam, respectivamente, as deformações de membrana e flexão, e $\boldsymbol{\varepsilon}^{c}$ é o vetor das deformações não-lineares que traduz o acoplamento entre os efeitos de membrana e flexão no plano médio. Além disso, ele não aparece na modelagem linear de placas finas tal que os efeitos de flexão e membrana podem ser resolvidos separadamente. Entretanto, para o caso não-linear, isto não é mais possível sendo necessário considerar o acoplamento.

Considerando que os campos de deslocamentos são discretizados em elementos finitos por funções de interpolação para um elemento de placa plana retangular, os componentes de deformação de membrana, flexão e acoplamento entre elas pode-se escrever as componentes do tensor de Green-Lagrange, Eq.2, como:

$$
\boldsymbol{\varepsilon}^{m}=\boldsymbol{B}^{m} \tilde{\boldsymbol{u}}(t), \boldsymbol{\varepsilon}^{c}=\frac{1}{2} \boldsymbol{B}^{n l}(\tilde{\boldsymbol{w}}) \tilde{\boldsymbol{w}}(t), \boldsymbol{K}^{f}=\boldsymbol{B}^{f} \tilde{\boldsymbol{w}}(t)
$$

onde $\tilde{\boldsymbol{u}}(t)=\left[\begin{array}{ll}u & v\end{array}\right]^{T}, \tilde{\boldsymbol{w}}(t)=\left[\begin{array}{lll}w & \psi_{x} & \psi_{y}\end{array}\right]^{T}$, e as matrizes $\boldsymbol{B}^{m}, \boldsymbol{B}^{n l}$ e $\boldsymbol{B}^{f}$ são formadas pelos operados diferenciais das relações deformações-deslocamentos para um elemento de placa plana retangular formada por quatro nós e cinco graus de liberdade por nós.

A partir dessas relações, pode-se escrever o princípio dos trabalhos virtuais, em que são levadas em consideração a energia cinética e a energia potencial de deformação, como descrito abaixo:

$$
\delta \prod=\delta \int_{t_{1}}^{t_{2}}\left(U_{0}+T_{0}\right) d t=0
$$

Sendo que $T_{0}$ é a energia cinética do sistema, utilizada para a formulação da matriz de massa relacionada ao sistema e a energia potencial $U_{0}$ é a soma das energias potencias das forças externas impostas e a energia potencial de deformação, que é dada por:

$$
U_{d e f}=\frac{1}{2} \int_{\Omega} E \dot{S} d V
$$

Onde S é o tensor de Piolla-Kirchhoff.

A partir da formulação variacional do tensor de Green-Lagrange, então, é possível obter a matriz de rigidez do sistema:

$$
\boldsymbol{K}^{(e)}=\boldsymbol{K}_{l}^{(e)}+\Delta \boldsymbol{K}_{n l}^{(e)}(\tilde{\boldsymbol{w}})
$$


Nota-se que a matriz de rigidez têm duas componentes, o primeiro termo correspondente á contribuição linear e o segundo a contribuição não-linear que diz respeito ao acoplamento entre os efeitos de flexão e membrana, que são calculadas por:

$$
\begin{gathered}
\boldsymbol{K}_{l}^{(e)}=\sum_{k=1}^{n} \int_{z=z_{k}}^{z_{k+1}} \int_{y=0}^{y=b} \int_{x=0}^{y=a}\left[\begin{array}{cc}
\boldsymbol{B}^{m^{T}} \boldsymbol{C}^{m}\left(\theta_{k}\right) \boldsymbol{B}^{m} & \boldsymbol{0} \\
\boldsymbol{0} & \boldsymbol{B}^{f^{T}} \boldsymbol{C}^{f}\left(\theta_{k}\right) \boldsymbol{B}^{f}
\end{array}\right] d x d y d z \\
\Delta \boldsymbol{K}_{n l}^{(e)}(\tilde{\boldsymbol{w}})=\sum_{k=1}^{n} \int_{z=z_{k}}^{z_{k+1}} \int_{y=0}^{y=b x=a} \int_{x=0}^{x=a}\left[\begin{array}{cc}
\frac{1}{2} \boldsymbol{B}^{m^{T}} \boldsymbol{C}^{m}\left(\theta_{k}\right) \boldsymbol{B}^{n l}(\tilde{\boldsymbol{w}}) \\
\boldsymbol{B}^{n l^{T}}(\tilde{\boldsymbol{w}}) \boldsymbol{C}^{m}\left(\theta_{k}\right) \boldsymbol{B}^{m} & \frac{1}{2} \boldsymbol{B}^{n l^{T}}(\tilde{\boldsymbol{w}}) \boldsymbol{C}^{m}\left(\theta_{k}\right) \boldsymbol{B}^{n l}(\tilde{\boldsymbol{w}})
\end{array}\right] d x d y d z
\end{gathered}
$$

Após a obtenção das matrizes de massa e rigidez, é possível escrever as equações do movimento global do sistema, no domínio do tempo, da forma matricial:

$$
\left[\begin{array}{cc}
\boldsymbol{M}_{m} & \boldsymbol{0} \\
\boldsymbol{0} & \boldsymbol{M}_{f}
\end{array}\right]\left\{\begin{array}{l}
\ddot{\tilde{\boldsymbol{u}}}(t) \\
\ddot{\tilde{\boldsymbol{w}}}(t)
\end{array}\right\}+\left(\left[\begin{array}{cc}
\boldsymbol{K}_{m} & \boldsymbol{0} \\
\boldsymbol{0} & \boldsymbol{K}_{f}
\end{array}\right]+\left[\begin{array}{cc}
\boldsymbol{0} & \frac{1}{2} \Delta \boldsymbol{K}_{1} \\
\Delta \boldsymbol{K}_{1}^{T} & \Delta \boldsymbol{K}_{2}
\end{array}\right]\right)\left\{\begin{array}{l}
\tilde{\boldsymbol{u}}(t) \\
\tilde{\boldsymbol{w}}(t)
\end{array}\right\}=\left\{\begin{array}{l}
\boldsymbol{f}^{\tilde{\boldsymbol{u}}}(t) \\
\boldsymbol{f}^{\tilde{\boldsymbol{w}}}(t)
\end{array}\right\}
$$

Ou ainda:

$$
\boldsymbol{M} \ddot{\boldsymbol{u}}(t)+\underbrace{\boldsymbol{K}(\boldsymbol{u}) \boldsymbol{u}(t)}_{\boldsymbol{f}_{n l}}-\boldsymbol{f}(t)=0
$$

Na Eq.(10), nota-se a presença de uma força não linear que pode ser entendida como uma perturbação no comportamento do sistema quando ele é submetido a um grande deslocamento.

\subsection{Resolução da equação do movimento pelo método de Newmark}

O método de Newmark, utilizado para a resolução de equações diferenciais de segunda ordem, foi empregado neste trabalho para a resolução da equação do movimento da placa (Eq.10).

Sendo $\boldsymbol{u}_{\boldsymbol{i}}=\left[\tilde{\boldsymbol{u}}_{\boldsymbol{i}}(t) \tilde{\boldsymbol{w}}_{\boldsymbol{i}}(t)\right]$ expandindo as variáveis de estado $\boldsymbol{u}_{\boldsymbol{i}}$ (deslocamento), $\dot{\boldsymbol{u}}_{\boldsymbol{i}}$ (velocidade) em série de Taylor e truncando no termo de $3^{\mathrm{a}}$ ordem temos:

$$
\begin{gathered}
u_{i}=u_{i-1}+\Delta t \dot{u}_{i-1}+\frac{\Delta t^{2} \ddot{u}_{i-1}}{2}+\frac{\Delta t^{3} \dddot{u}_{i-1}}{6} \\
\dot{u}_{i}=\dot{u}_{i-1}+\Delta t \ddot{u}_{i-1}+\frac{\Delta t^{2} \dddot{u}_{i-1}}{2}
\end{gathered}
$$

Sendo $\Delta \boldsymbol{t}=\boldsymbol{t}_{\boldsymbol{i}}-\boldsymbol{t}_{\boldsymbol{i}-1}$ o passo de tempo.

Inserindo os parâmetros $\gamma$ e $\beta$ iguais a $1 / 2$ e $1 / 6$, as relações das variáveis de estado passam a corresponder ao método de aceleração linear. (BATHE,1996). Sendo assim pode-se escrever $\dddot{\boldsymbol{u}}_{i-1}$, tal que:

$$
\dddot{u}_{i-1}=\frac{\ddot{u}_{i}-\ddot{u}_{i-1}}{\Delta t}
$$

Substituindo esta relação nas Eq.2.10 e Eq.2.11, chegamos às expressões:

$$
\begin{gathered}
\boldsymbol{u}_{i}=\boldsymbol{u}_{i-1}+\Delta t \dot{\boldsymbol{u}}_{i-1}+\ddot{u}_{i-1} \Delta t^{2}\left(\frac{1}{2}-\beta\right)+\beta \Delta t^{2} \ddot{u}_{i} \\
\dot{\boldsymbol{u}}_{i}=\dot{\boldsymbol{u}}_{i-1}+\Delta t \ddot{u}_{i-1}(1-\gamma)+\gamma \Delta t \ddot{u}_{i}
\end{gathered}
$$


Isolando o termo $\ddot{\boldsymbol{u}}_{i}$ na eq.2.14 em função de $\boldsymbol{u}_{i}$ e substituindo na eq.2.15 obtêm-se as equações relacionadas à aceleração $\left(\ddot{\boldsymbol{u}}_{\boldsymbol{i}}\right)$ e à velocidade $\left(\dot{\boldsymbol{u}}_{\boldsymbol{i}}\right)$ em função dos deslocamentos desconhecidos $\left(\boldsymbol{u}_{\boldsymbol{i}}\right)$. Estas equações, quando substituídas na Eq.(16) resultam em um problema quase-estático, como é mostrado na Eq. (17).

$$
\begin{gathered}
\boldsymbol{M} \ddot{\boldsymbol{u}}_{\boldsymbol{i}}+\boldsymbol{C} \dot{\boldsymbol{u}}_{\boldsymbol{i}}+\boldsymbol{K} \boldsymbol{u}_{\boldsymbol{i}}=\boldsymbol{F}_{\boldsymbol{i}} \\
\hat{\boldsymbol{K}}\left(\boldsymbol{u}_{\boldsymbol{i}-1}\right) \Delta \boldsymbol{U}_{\boldsymbol{i}}=\hat{\boldsymbol{f}}
\end{gathered}
$$

Onde, $\mathrm{M}$ representa a massa do sistema, $\mathrm{C}$ o amortecimento, $\mathrm{K}$ a rigidez, e, $\mathrm{F}_{\mathrm{i}}$ a força externa aplicada.

$\hat{\boldsymbol{K}}$ é a matriz de rigidez efetiva, que é dada por:

$$
\hat{K}=K^{t}+\frac{\gamma}{\beta \Delta t} \boldsymbol{C}+\frac{1}{\beta \Delta t^{2}} \boldsymbol{M}
$$

$\Delta \boldsymbol{U}_{i}$ é a variação do deslocamento no intervalo entre $\mathrm{t}_{\mathrm{i}}$ e $\mathrm{t}_{\mathrm{i}-1}$, ou seja, $\Delta \boldsymbol{U}_{i}=\boldsymbol{U}_{\boldsymbol{i}}-\boldsymbol{U}_{\boldsymbol{i}-1}$.

E, $\hat{f}$ é uma força dinâmica instantânea aplicada, dada por:

$$
\hat{\boldsymbol{f}}=\boldsymbol{F}_{\boldsymbol{i}}-\boldsymbol{F}_{i-1}+\left(\frac{1}{\beta \Delta \boldsymbol{t}} \boldsymbol{M}+\frac{\gamma}{\beta} \boldsymbol{C}\right) \dot{\boldsymbol{u}}_{\boldsymbol{i}-1}+\left(\frac{1}{2 \beta} \boldsymbol{M}+\left(\frac{\gamma}{2 \beta}-1\right) \Delta \boldsymbol{t} \boldsymbol{C}\right) \ddot{\boldsymbol{u}}_{\boldsymbol{i}-1}
$$

A Eq. (17) pode ser obtida pela aproximação da força não-linear para o ponto $t_{i}$ pela expansão em série de Taylor em torno do deslocamento no tempo $t_{i-1}$. Ou seja

$$
f_{n l}\left(\mathbf{u}_{i}\right)=K\left(\mathbf{u}_{i}\right) \mathbf{u}_{i}=f_{n l}\left(\mathbf{u}_{i-1}\right)+\frac{\partial f_{n l}}{\partial u}\left(\mathbf{u}_{i}-u_{i-1}\right)
$$

Onde $\frac{\partial \boldsymbol{f}_{n l}}{\partial \boldsymbol{u}}$ representa a derivada da força não-linear em função do deslocamento. E, para cada instante de tempo é representada pela matriz de rigidez tangente.

\subsection{Desenvolvimento da matriz tangente}

Como já foi exposto anteriormente, a matriz de rigidez tangente $\left(K^{t}\right)$, é a forma discreta da derivada da força nãolinear em relação ao deslocamento no tempo $t_{i}$. Essa matriz, ou gradiente espacial da força não-linear, é necessária para a integração da equação do movimento da placa pelo método de Newmark.

A força não-linear pode ser escrita como sendo composta por componentes da força na superfície da placa e as forças que atuam na flexão. Sendo assim, pode-se escrever:

$$
f_{n l}=\left\{\begin{array}{l}
f^{\tilde{u}} \\
f^{\tilde{w}}
\end{array}\right\}
$$

E, então, escreve-se o gradiente espacial da força não-linear, ou seja:

$$
\boldsymbol{K}^{t}=\left[\begin{array}{ll}
\frac{\partial \boldsymbol{f}^{\tilde{u}}}{\partial \tilde{\boldsymbol{u}}} & \frac{\partial \boldsymbol{f}^{\tilde{u}}}{\partial \tilde{\boldsymbol{w}}} \\
\frac{\partial \boldsymbol{f}^{\tilde{w}}}{\partial \tilde{\boldsymbol{u}}} & \frac{\partial \boldsymbol{f}^{\tilde{w}}}{\partial \tilde{\boldsymbol{w}}}
\end{array}\right]
$$

Escrevendo a derivada da força não-linear como:

$$
d f_{n l}(\mathbf{u})=d f^{\tilde{u}}(\mathbf{u})+d f^{\tilde{w}}(\mathbf{u})
$$

E, então, desenvolvendo as derivadas das rigidezes multiplicadas pelos graus de liberdade, como mostrado na Eq.9, chega-se aos componentes da matriz de rigidez tangente:

$$
\boldsymbol{K}^{t}=\boldsymbol{K}^{l}+\Delta \boldsymbol{K}^{n l}+\Delta \boldsymbol{K}^{t}
$$

Sendo o primeiro termo a contribuição linear e o segundo a contribuição não-linear, já mostradas nas Eq. (7) e Eq.(8). E o último termo é dado por: 


$$
\Delta \boldsymbol{K}^{t}=\sum_{k=1}^{n} \int_{z=z_{k}}^{z_{k+1}} \int_{\boldsymbol{y}=0}^{y=b} \int_{x=0}^{\boldsymbol{x}=\boldsymbol{a}}\left[\begin{array}{cc}
\boldsymbol{0} & \frac{1}{2} \boldsymbol{B}^{\boldsymbol{m}^{T}} \boldsymbol{C}^{\boldsymbol{m}}\left(\theta_{k}\right) \boldsymbol{B}^{n l}(\tilde{\boldsymbol{w}}) \\
0 & \frac{1}{2} \boldsymbol{B}^{n l^{T}}(\tilde{\boldsymbol{w}}) \boldsymbol{C}^{m}\left(\theta_{k}\right) \boldsymbol{B}^{n l}(\tilde{\boldsymbol{w}})+\boldsymbol{G}^{T} \boldsymbol{T}^{m} \boldsymbol{G}
\end{array}\right] \boldsymbol{d x d y d z}
$$

Onde $\boldsymbol{G}$ é a matriz de derivadas das relações de deformação-deslocamento relacionadas à flexão e $\boldsymbol{T}^{\boldsymbol{m}}$ é dado pela relação : $\boldsymbol{D}^{m} \boldsymbol{E}^{m}$.

\section{SIMULAÇÕES NUMÈRICAS}

Com o objetivo de validar o modelo e observar o comportamento da placa que é submetida a grandes deslocamentos, foi imposta uma força harmônica pontual da forma $F_{e}(t)=F_{0} \operatorname{sen}(\omega t)$, em que $F_{0}$ representa a amplitude da força imposta e a frequência de excitação é igual à primeira frequência natural da placa, é aplicada no centro da dimensão oposta ao engaste. A Fig. 1 mostra a placa engastada-livre discretizada em 10 elementos ao longo do seu comprimento e 10 ao longo de sua largura, cujas características físicas e geométricas são mostradas na Tab. 1 .

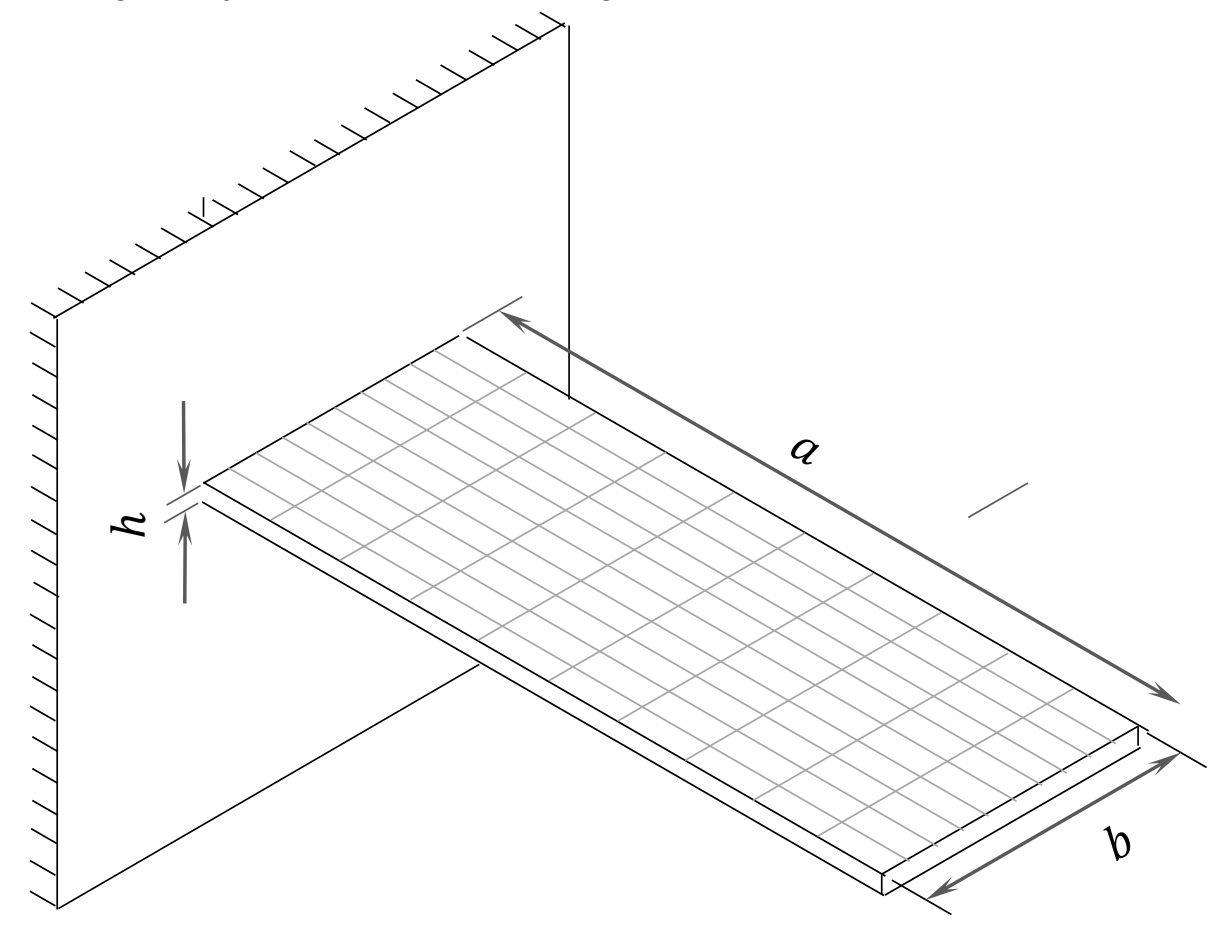

Figura 1. Representação esquemática de uma placa discretizada em elementos finitos

Tabela 4.2 - Propriedades Físicas e Geométricas da placa

\begin{tabular}{|c|c|c|}
\hline \multicolumn{2}{|c|}{ PLACA } \\
\hline \multirow{3}{*}{ Propriedades Geométricas } & Comprimento [m] & 0.2 \\
\cline { 2 - 3 } & Largura [m] & 0.05 \\
\cline { 2 - 3 } & Espessura [m] & 0.002 \\
\hline \multirow{3}{*}{ Propriedades Físicas } & Densidade Volumétrica $\left[\mathrm{Kg} / \mathrm{m}^{3}\right]$ & 7800 \\
\cline { 2 - 3 } & Módulo de Elasticidade de Young & $2.1 \mathrm{e} 5$ \\
\cline { 2 - 3 } & {$[\mathrm{MPa}]$} & 0.3 \\
\hline
\end{tabular}

Para a resolução das equações do movimento foi utilizado o método de integração de Newmark juntamente com o método de Newton-Raphson, com o objetivo de realizar correções na resolução feita pelo método integrador devido às características não-lineares do sistema. O intervalo de tempo analisado é de 0 a 4 segundos com passo de tempo igual a $0.0005 \mathrm{~s}$. 
A Figura 2, exibe a respostas temporal e o plano de fase do sistema para uma força senoidal com amplitude de $8 \mathrm{~N}$. A resposta no domínio do tempo, apresenta poucas características não-lineares em suas amplitudes o que também pode ser observado no seu plano de fase.

.À medida em que aumentamos a amplitude da força, para 40N (Fig. 3) e consequentemente o deslocamento da placa, observa-se uma discreta mudança nos picos das amplitudes das respostas o que sugere o surgimento do comportamento não-linear do sistema.

As respostas obtidas para $80 \mathrm{~N}$ e $200 \mathrm{~N}$, as não-linearidades tornam-se mais evidentes tanto no domínio do tempo, quanto no plano de fase além de serem demonstrados pontos de instabilidade que surgem no sistema devido às não linearidades.
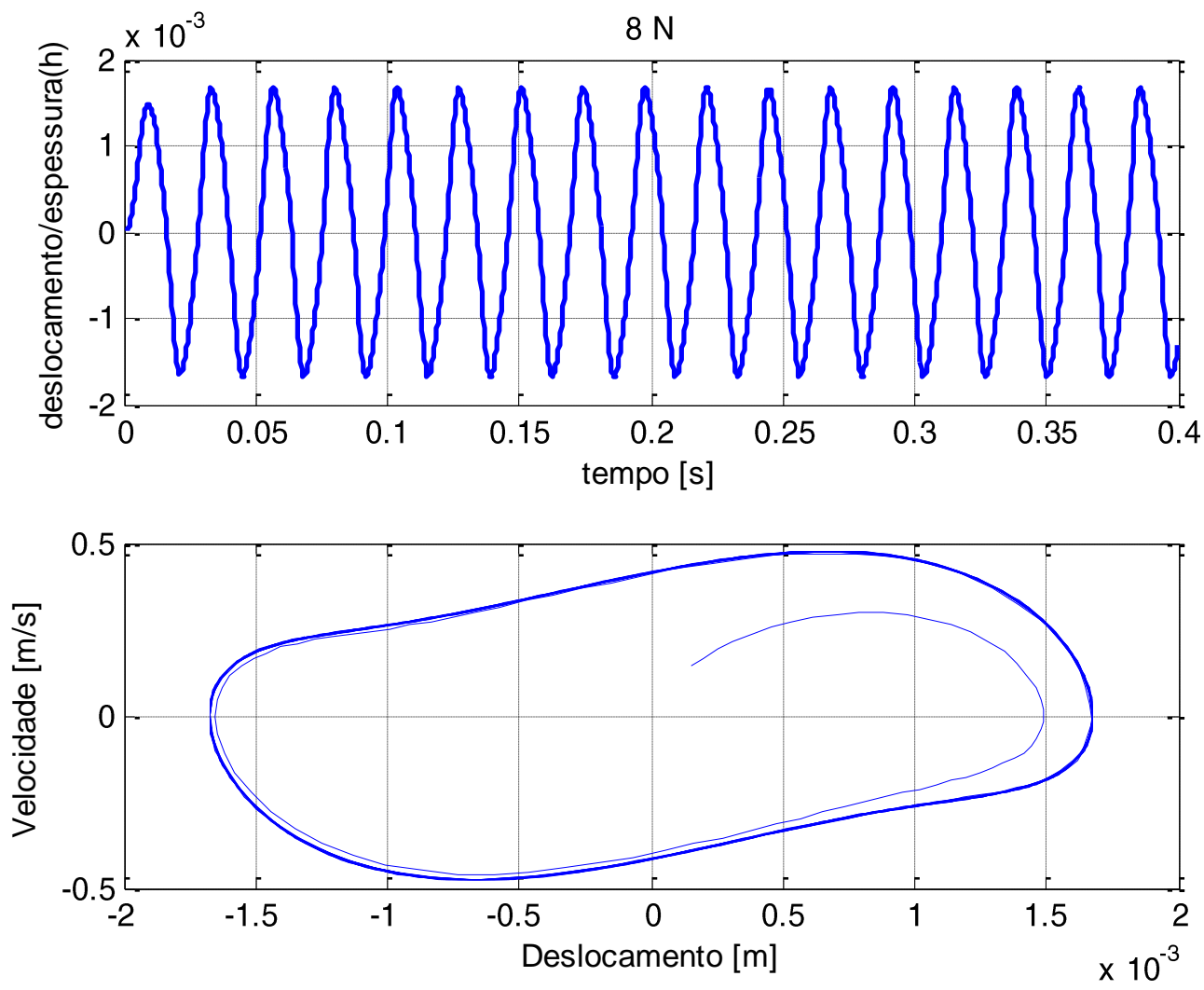

Figura 2. Resposta temporal e plano de fase da placa para uma força de $8 \mathrm{~N}$ 

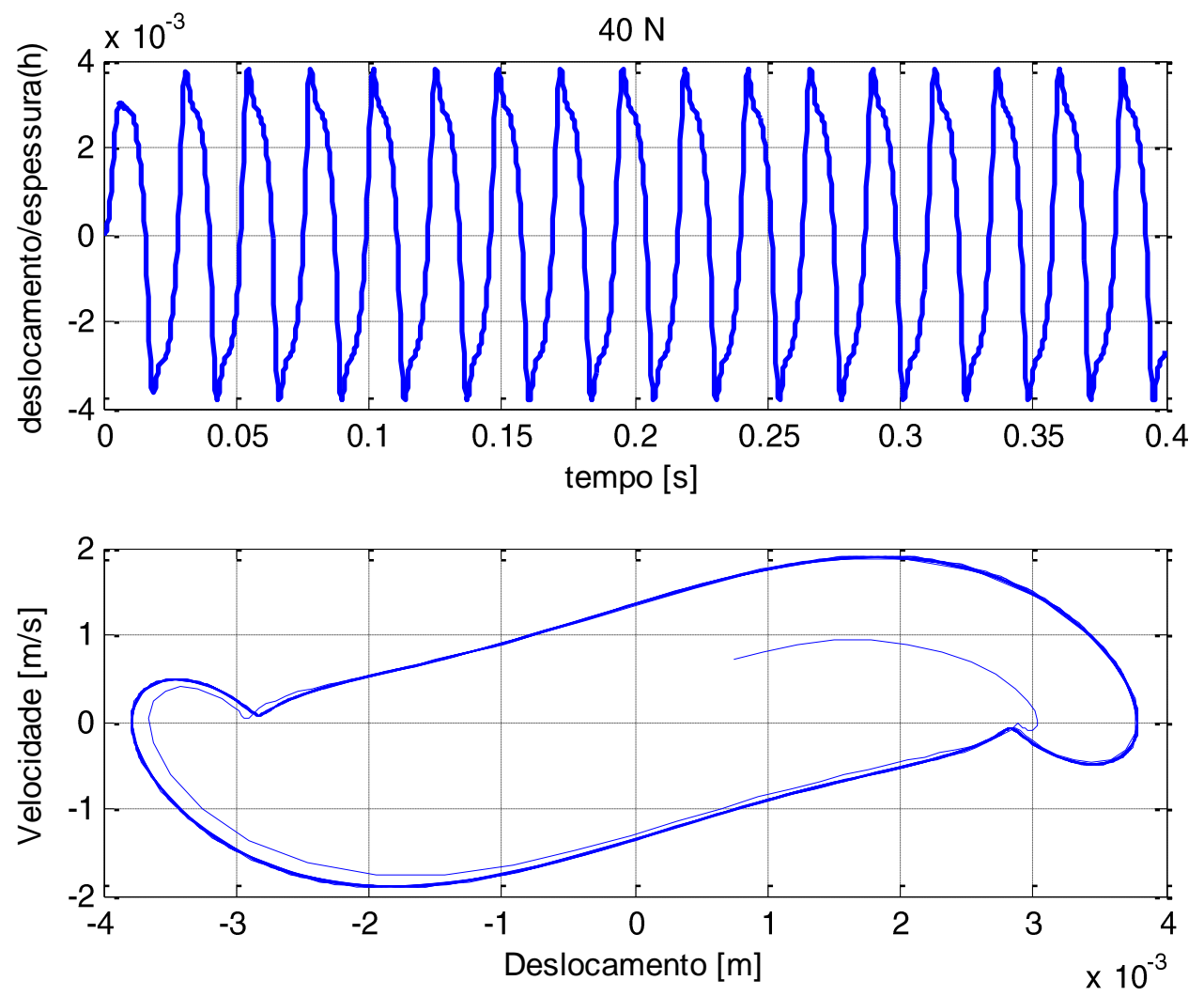

Figura 3. Resposta temporal e plano de fase da placa para uma força de $40 \mathrm{~N}$
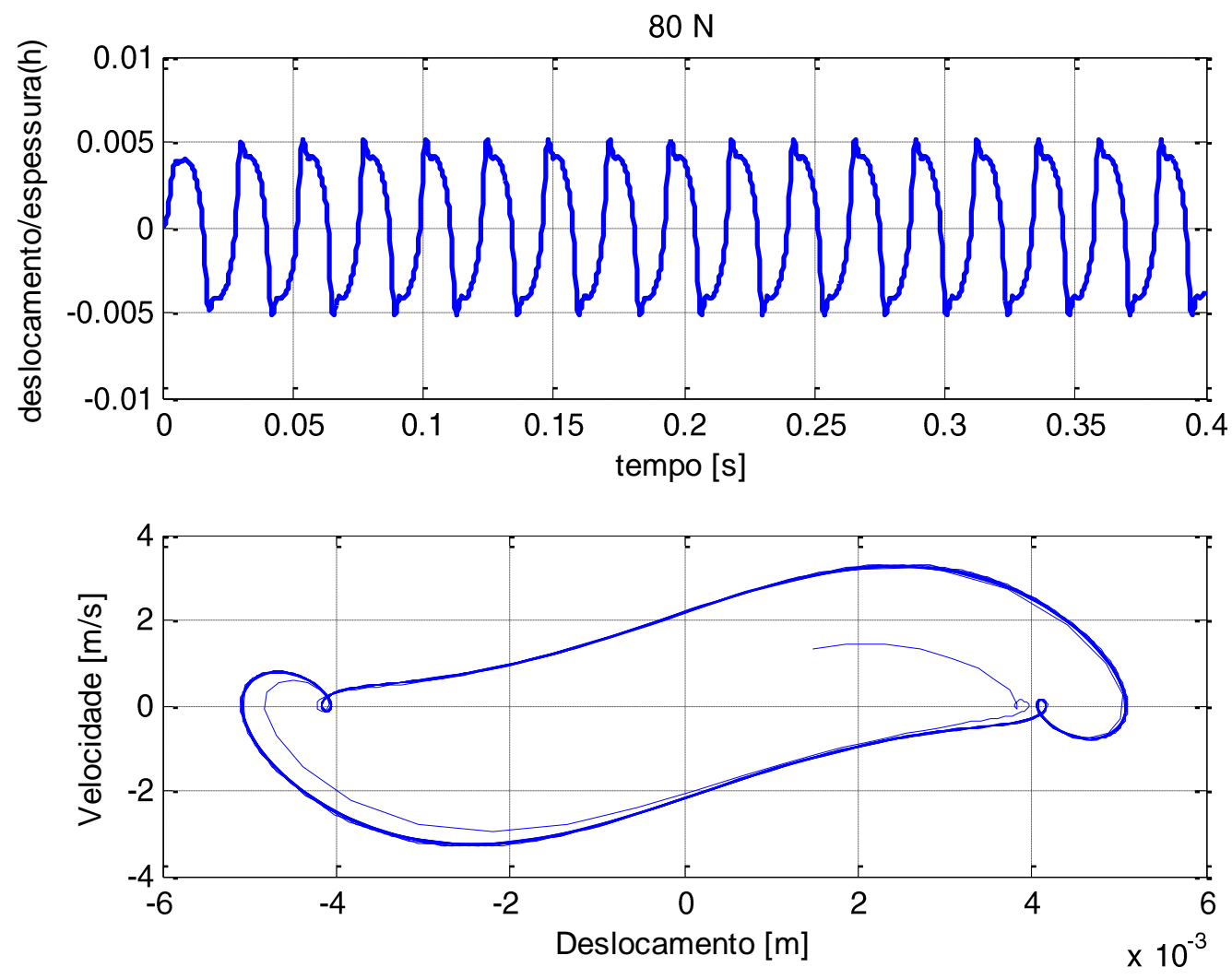

Figura 4. Resposta temporal e plano de fase da placa para uma força de $80 \mathrm{~N}$ 

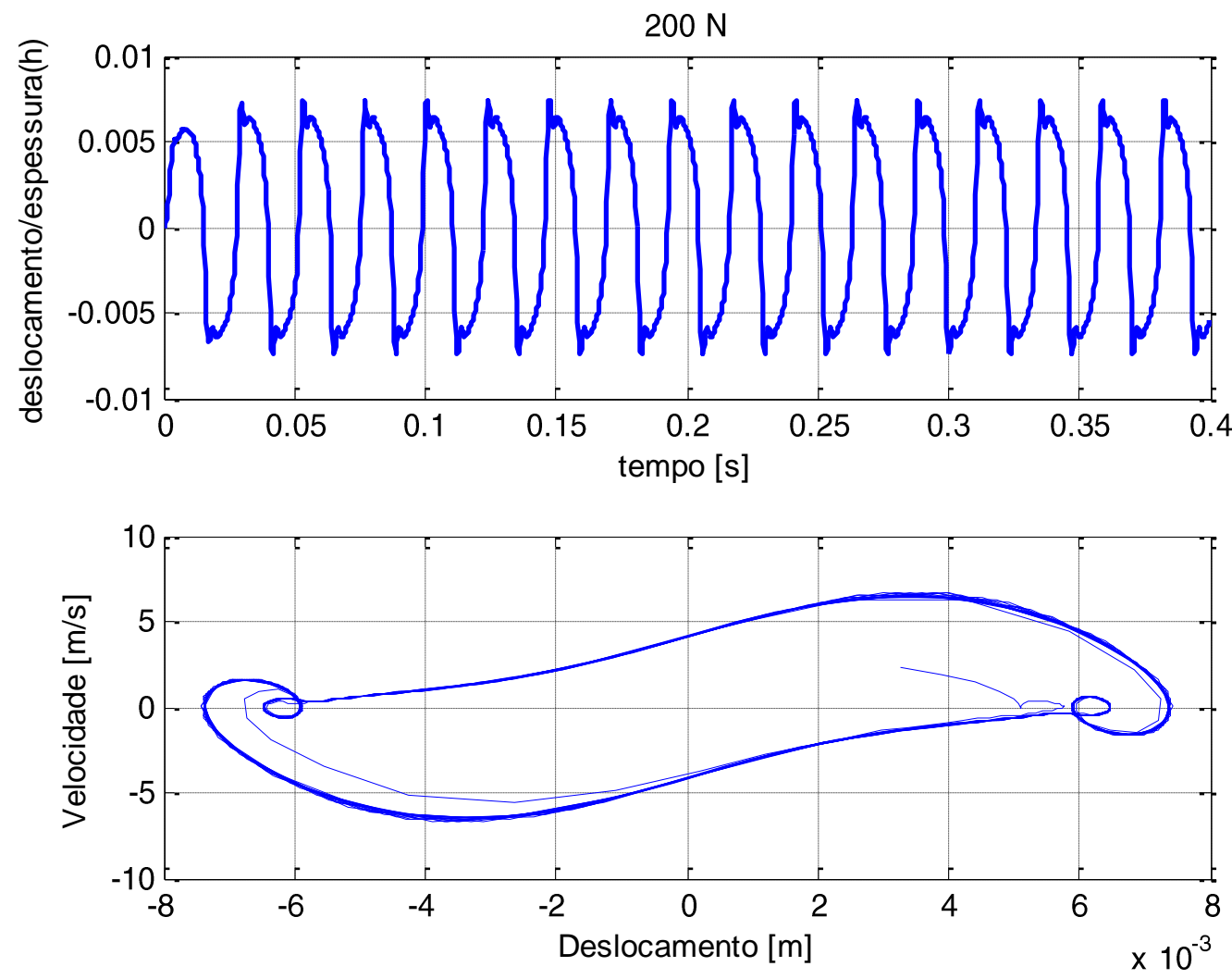

Figura 5. Resposta temporal e plano de fase da placa para uma força de $200 \mathrm{~N}$

\section{Conclusões}

Neste trabalho foi realizada uma simulação numérico-computacional de placas em grandes deslocamentos e rotações moderadas, o que nos leva à uma formulação não-linear. Foram desenvolvidas e mostradas o equacionamento utilizado para a obtenção das respostas de um sistema a partir desta formulação.

Com os resultados obtidos a partir das simulações nota-se a importância de um estudo em que são levadas em consideração o efeito de acoplamento entre os efeitos de membrana e flexão da viga, que são as fontes de não-linearidades levadas em consideração neste estudo, sendo que, à medida em que a amplitude da força aplicada ao sistema as nãolinearidades tornam-se cada vez mais evidentes.

\section{BIBLIOGRAFIA}

Bathe, K.-J. Finite Element Procedures. 1.ed. Prentice Hall, 1996. 1037p

Gerges, Y. 2013.Méthodes de réduction de modèles em vibroacoustique non-linéaire.162f.

Tese de Doutorado - Université de Franche - Comté.

Moussaoui, F.;Benamar, R. 2002.Non-Linear Vibrations of Shell-Type Structures: A Riview with Bibliography. Journal of Sound and Vibration. v. 255, n 1, p.161-184.

Zienkiewicz, O.C.; Taylor, R.L. 2000. The Finite Element Method: Solid Mechanics,

Volume 2. 5. Ed. Butterworth-Heinemann. 459 p.

\section{RESPONSABILIDADE AUTORAL}

$\mathrm{O}(\mathrm{s})$ autor(es) é(são) o(s) único(s) responsável(is) pelo conteúdo deste trabalho 